\title{
The Real-Time Look-Ahead NURBS Curve Interpolation Algorithm
}

\author{
Gengzhu Wang \\ University of Science and Technology Liaoning, Anshan, Liaoning, 114051, China \\ wgz20203@sina.com
}

Keywords: Look-ahead, Interpolation algorithm, Numerical control system

Abstract : To ensure a given chord error, through the division of the cusp, the NURBS (Non-Uniform Rational B-Splins) curve is divided into several sections and the speed of the various sections is planned accordingly. The acceleration and deceleration time period is recalculated, which results in a smooth speed transition curve.

\section{Trajectory Look-Ahead}

Paper [1] presents a NURBS curve interpolation algorithm with dynamic adaptive ability and curve look-ahead control ability. The algorithm is adaptive to machine, having high contour machining precision, the feed rate can be adaptively adjusted to the change of curvature, the feed rate fluctuation is small, which not only can ensure the surface quality of the work piece, but also can avoid causing excessive impact on the machine.

In actual processing, only when the initial velocity and terminal velocity are correctly planned, the smooth program transition rate between segments can be guaranteed. Therefore, the movement control system must address a number of blocks in advance to identify the turning point of sharp turns, and timely sending out the deceleration signal. The function that the numerical control system must have is the pre-process trajectory control, which is also known as Look-ahead ${ }^{[23.4]}$.

The trajectory look-ahead control or speed look-ahead control is a key technique of numerical control system. If there are sharp changes in the feeding direction, the feeding track monitoring system will be slow down in some way. If the change in the direction of feeding is smooth, the system will rapidly increase the feed rate to the maximum programming speed.

\section{Determine the number of look-ahead segments}

Using a complete "accelerated, uniform, deceleration" on the non interpolated curve as the interpolator look-ahead segment length, that is, it is to be determined by the seven mode model reference length of the five sections of S Curve acceleration and deceleration model.

Taking into account the extreme situation, when the initial and terminal speed $V_{\text {str }}=V_{\text {end }}=0$, so the required look-ahead curve length is

$$
L_{r e f}=2 F \sqrt{\frac{F}{J_{\max }}}
$$

Where, $F$ is the feed rate. $J_{\max }$ is the jerk.

\section{NURBS interpolation algorithm with the look-ahead real-time}

Cusp detection. There are two criteria to detect the cusp ${ }^{[5]}$.

The first criterion to determine the cusp is: at the cusp, the derivative of the curvature is 0 . The formula is as follows.

$$
\left.\frac{d k(u)}{d t}\right|_{u=u_{k}}=0
$$


Where, $u_{k}$ is the node vector of the possible cusp. $k(u)$ is the curvature, it is calculated as.

$$
k(u)=\frac{\left|p^{\prime}(u) p^{\prime \prime}(u)\right|}{\left|p^{\prime}(u)\right|^{3}}
$$

The second cusp identification criterion is: the curvature of cusp exceeds the limit $k_{t h}$. The curvature limits $k_{t h}$. is calculated as follows.

$$
k_{t h}=\frac{A_{\max }}{F^{2}}
$$

Only the point meets the above mentioned two criteria will be identified as the cusp.

Calculation the cumulative segment length. The cumulative segment length $L^{c}(1,2, \cdots, m)$ can be calculated.

$$
\begin{aligned}
& L^{c}=\sum_{j=1}^{c} L_{\text {seg }}^{j}(C=1,2, \ldots ., m) \\
& L_{\text {seg }}^{m}=\sum_{i=1}^{n}\left|p\left(u_{i+1}\right)-p\left(u_{i}\right)\right|
\end{aligned}
$$

After the length of each segment $L_{\text {seg }}{ }^{m}$ is obtained, the calculation of the cumulative segment length $L^{c}(1,2, \cdots, m)$ is very easy.

The adaptive feed rate based on the chord error limit. The real-time NURBS interpolation uses the straight line to approximate the curve, so it is inevitable that there is arc chord error ER. It requires the system to conduct the real-time dynamic adjustment to feed rate during the real-time interpolation to ensure high contour accuracy during the high-speed processing.

By the geometry relationship shown in Fig.1, ER can be calculated.

$$
E R=\rho_{i}-\sqrt{\rho_{i}-\left(\frac{L_{i}}{2}\right)^{2}}
$$

Because $\mathrm{L}_{\mathrm{i}}=\mathrm{V}\left(\mathrm{u}_{\mathrm{i}}\right) \mathrm{T}$, so

$$
E R=\rho_{i}-\sqrt{\rho_{i}-\left(\frac{V\left(u_{i}\right) T}{2}\right)^{2}}
$$

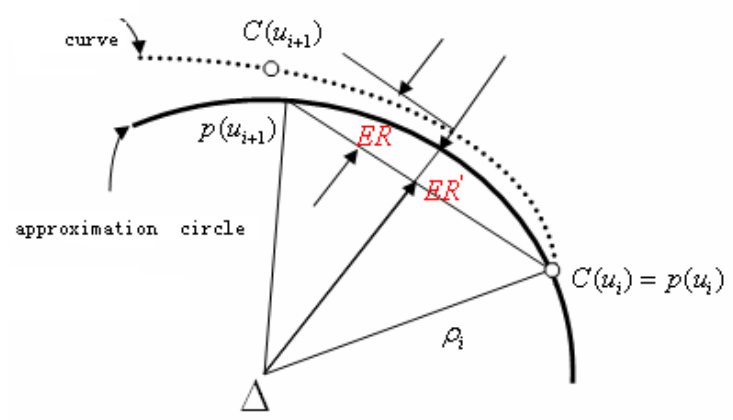

Fig. 1 The chord error

It can be seen that the chord error is related to the length of every step and the interpolation curvature. When the actual error is within the allowable range, still to calculate the interpolation step according to the given feed rate; if the actual error exceeds the allowable range, the actual processing speed must be reduced, and to calculate the interpolation step in accordance with the required tolerance. Therefore, the adaptive feed rate $V_{a f}$ based on the chord error is introduced, the formula is

$$
V_{a f}= \begin{cases}F & \text { if } \frac{2}{T} \sqrt{\rho_{i}^{2}-\left(\rho_{i}-E R\right)^{2}}>F \\ \frac{2}{T} \sqrt{\rho_{i}^{2}-\left(\rho_{i}-E R\right)^{2}} & \text { if } \frac{2}{T} \sqrt{\rho_{i}^{2}-\left(\rho_{i}-E R\right)^{2}} \leq F\end{cases}
$$


Where, $\rho_{i}=\frac{1}{k_{i}}$, after obtaining the curvature. $\rho_{i}$ can be known, the formula to calculate the curvature is shown in Fig 1.

Calculation the adaptive feed rate on the basis of curvature. The curvature-based feed rate is calculated as follows.

$$
V_{c b f}\left(u_{i}\right)=\frac{k_{c b c}}{k\left(u_{i}\right)+k_{c b c}} F
$$

Where, $k_{c b c}$ is the given curvature value maintaining the continuous derivative of $V_{c b f}$, when $K\left(u_{i}\right)=0$, $V_{c b f}=$ F. $K\left(u_{i}\right)$ is the curvature at points $u_{\mathrm{i}}$ of the NURBS. $F$ is the given feed rate of the NC program. The feed speed at a given point on the curve is:

$$
V\left(u_{i}\right)=\max \left\{\min \left[V_{a f}\left(u_{i}\right), V_{c b f}\left(u_{i}\right), F_{\max }\right], F_{\min }\right\}
$$

Rate planning. For given limit conditions, such as: $A_{\max }, J_{\max }, F$, after the calculation of the parameters $L_{s e g}{ }^{m}, V_{\text {str }}, V_{\text {end }}, V_{\max }$ of each sub-section, corresponding to the control S-curve acceleration and deceleration curve, to compute the rest of parameters of acceleration and deceleration $T_{\text {str }}, T_{\text {end }}, T_{c}, J_{\text {str }}, J_{\text {end }}$. The five parameters will be used in the next step real-time interpolation process.

After comprehensive consideration, the feed rate of deceleration methods is specified into the following 7 types, as shown in Figure 2.

Then in connection with the different types, the corresponding solution is used to plan the trajectory of the segment. The specific process is given as follows:

Calculation the length of $L_{r 1}, L_{r 2}, L_{r 3}, L_{r 4}$.

$$
\begin{aligned}
& L_{r 1}=2 V_{s t r} T \\
& L_{r 2}=\left(V_{s t r}+V_{\text {end }}\right) \sqrt{\frac{\left|V_{\text {end }}-V_{\text {str }}\right|}{J_{\text {max }}}} \\
& L_{r 3}=\left(V_{s t r}+V_{\text {max }}\right) \sqrt{\frac{V_{\text {max }}-V_{s t r}}{J_{\text {max }}}}+\left(V_{\text {max }}+V_{\text {end }}\right) \sqrt{\frac{V_{\text {max }}-V_{\text {end }}}{J_{\text {max }}}} \\
& L_{r 4}=\left(V_{s t r}+V_{\text {max }}\right) \sqrt{\frac{V_{\text {max }}-V_{s t r}}{J_{\text {max }}}}
\end{aligned}
$$

where, $L_{r 1}$ is the minimum required length of $I I$, III. $L_{r 2}$ is the minimum required length that can be planned into the type VI. $L_{r 3}$ is the minimum required length that can be planned into the type VII; when $\left(V_{s t r}=V_{\max }\right)$ or $\left(V_{\text {end }}=V_{\max }\right) . L_{r 4}$ is the minimum required length that can be planned into the types $I V$ or $V$.

The parameter calculation method of acceleration and deceleration planning is as follows. Using the type $V I$ feed rate planning as an example. The curve that satisfies $V_{\text {str }} \neq V_{\max }, V_{\text {end }} \neq V_{\max }, L_{r 2} \leq L_{\text {seg }}^{m}<L_{r 3}$ can be classified as type $V I$. According to the type $V I$ feed rate features as shown in Figure 5, the following formula can be established. 

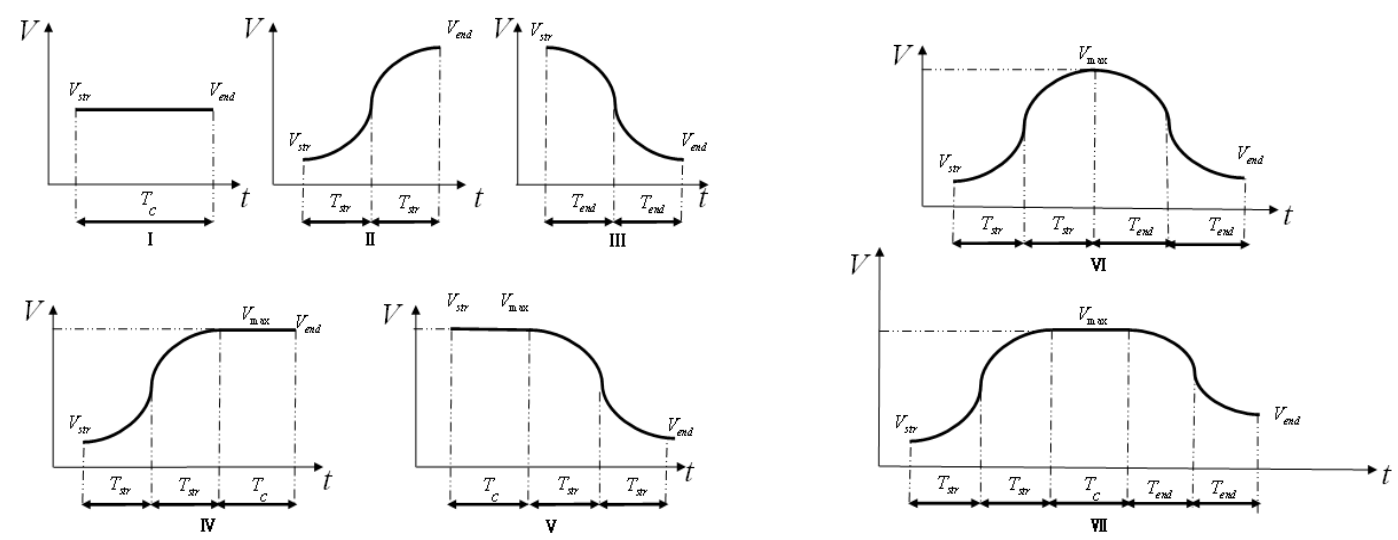

Fig.2 The types for the 5S-shape acceleration and deceleration control

$$
\begin{aligned}
& V_{\text {max }}=V_{\text {str }}+J_{\text {max }} T_{s t r}^{2}=V_{\text {end }}+J_{\max } T_{\text {end }}^{2} \\
& L_{\text {seg }}^{C}=\left(V_{\text {str }}+V_{\text {max }}\right) T_{\text {str }}+\left(V_{\text {str }}+V_{\text {max }}\right) T_{\text {end }} \\
& T_{\text {end }}=\sqrt{T_{\text {str }}+\frac{V_{\text {str }}-V_{\text {end }}}{J_{\text {max }}}}
\end{aligned}
$$

Substitute formula (16) and (18) into (17), obtain

$$
\begin{aligned}
& L_{s e g}^{c}=\left(2 V_{s t r}+J_{\max } T_{s t r}^{2}\right) T_{s t r}+\left(T_{s t r}+V_{\text {end }}+J_{\max } T_{s t r}^{2}\right) \sqrt{T_{s t r}+\frac{V_{s t r}-V_{\text {end }}}{J_{\max }}} \\
& f\left(T_{s t r}\right)=J_{\max }\left(V_{\text {end }}-V_{s t r}\right) T_{s t r}^{4}+2 J_{\max } L_{\text {seg }}^{C} T^{3}{ }_{s t r}-\left(V_{s t r}-V_{\text {end }}\right)^{2} T^{2}{ }_{s t r}
\end{aligned}
$$

Using Newton-Rapson iteration method to calculate $T_{s t r}$, the initial value is set as $T_{\max }=A_{\max } / J_{\max }$. $T_{\text {end }}$ is obtained by equation (18). Similarly, the parameters of other mode can be obtained.

\section{Summary}

In the adaptive process, the feed rate of the appropriate acceleration and deceleration method using speed and jerk constraint is re-planned. This article introduced a simplified calculation of model, and carried out a detailed analysis to the five-phased $\mathrm{S}$ curve acceleration and deceleration control method, the method can eliminate the feed velocity and acceleration sudden change phenomena during the process of feeding, and it also can improve the smoothness of the feeding movement, the interpolation trajectory obtained can guarantee a stable and no impact manufacturing process, which is helpful to improve manufacturing precision and quality.

\section{References}

[1] S.-S. Yeh, R.-L. Hsu. Adaptive-feedrate interpolation for parametric curves with a confined chord error. Computer Aided Design 2002,34:229-237

[2] Zhang Bolin, Xia Hongmei, Huang Xiaoming. Research and Application of High Speed CNC Machine Tools. China Mechanical Engineering.2001， 12(10):1132-1138

[3] Zhou Zhenggan, Li Heping, Wang Meiqing, Yang Min,Wu Xueli,. Methods for High Speed High Precision Machining. Journal of North China Institute of Technology.2000,21(4):345-348

[4] Schuett T J. A closer look at look-ahead speed and accuracy benefits .Modern Machine Shop, Mar, 1996, :20 26

[5] Ming-Tzong Lin, Meng-Shiun Tsai, Hong-Tzong Yau. Development of a dynamics-based NURBS interpolator with real-time look-ahead algorithm.International Journal of Machine Tools \& Manufacture.2007,(47):2246-2262 